\title{
Surgical Treatment of Radial Head Fractures: Review of Literature.
}

\author{
Edvin Selmani' ${ }^{1}$ Agron Dogjani²
}

\begin{abstract}
The aim of this systematic review was to search for and critically appraise articles directly comparing functional outcomes and complications for fixation (ORIF) versus arthroplasty for comminuted radial head fractures (Mason type 3) in adults.

Material and methods: A comprehensive study of Medline, Embase and Cochrane databases using specific search terms and limits was conducted. Strict eligibility criteria were applied to stringently screen resultant articles. Three comparative studies were identified and reviewed.

Results: Two studies found significantly better functional scores after replacement compared with ORIF in Mason type 3 fractures. The third study found no significant differences in functional score or range of motion, but did find that grip strength was better after ORIF. Complication rates were too heterogenous for conclusion.

Conclusion: Fixation with good reduction may be attempted in unstable Mason type 3 fractures, and arthroplasty may be considered if this is not possible. Further randomised comparative trials are required to clarify the decisionmaking between fixation and replacement. Functional outcomes and complications were conflicting in the studies included here. Ideally, treatment decision should take into account elbow stability and degree of comminution.
\end{abstract}

Key words: radial head fractures, fixation, arthroplasty, ORIF

*Corresponding author: Edvin Selmani

$\equiv$ E-mail: selmaniedvin@gmail.com

${ }^{1}$ University Hospital of Trauma, Tirana, Albania 


\section{Full Text}

\section{Introduction}

In orthopedic practice radial head and neck fractures account for $1.7 \%$ to $5.4 \%$ of all fractures seen[1]. They are the most common fractures involving the elbow[2,3] representing $33 \%$ of all elbow fractures[4]. $85 \%$ of radial head fractures occur in patients aged between 20 and 60 years[1]. Treatment is influenced by fracture characteristics such as fragment number, displacement, joint stability, and associated injuries[5]. Options include early motion, fragment excision, radial head excision, fixation, or replacement[2]. Studies of the optimal treatment of comminuted, unstable radial head fractures has been hindered by their relative infrequency and limited comparative data[6].

Radial head fractures usually result from a fall onto the outstretched hand with the elbow extended and forearm pronated[5]. Axial, valgus and postero-lateral rotational patterns of loading are responsible for these fractures[3]. The radial head plays an important role in elbow stability. Several radial head fracture classification systems have been developed. The Mason classification[7] is widely referenced to categorise radial head fractures[6]. Mason type 1 is an undisplaced fracture; type 2 displaced marginal fractures; and type 3 comminuted fractures involving the entire radial head. Johnston[8] also added type 4, which categorises radial head fracture with an ulno-humeral dislocation. Broberg and Morrey[9] further modified Mason's classification by including fractures of the radial neck and stratifying them based on articular segment displacement and fragment size. The Hotchkiss[10] modification includes clinical examination and provides treatment guidelines for radial head fractures.

The goal of treatment of radial head fractures is preservation of elbow stability, motion and maintenance of radial length[11]. There is a consensus that Mason type 1 fractures without mechanical block should be managed non-surgically with early active motion[5,12]. Good results have been reported in 86$100 \%$ patients with type 1 fractures[13]. Mason type 2, minimally displaced, isolated fractures with no block to motion may also be managed non-surgically with early active motion[12]. Studies have shown $85-95 \%$ good results[14]. Isolated Mason type 2 fractures with significant displacement or mechanical block should be reduced and internally fixed if possible[15]. Options for fixation include Herbert screws, K-wires, plates, fibrin glue and bio-absorbable pins[16]. 
Fragment excision may be considered if secure fixation cannot be achieved in patients with block to motion[2].

Early studies advocated excision of Mason type 2 and 3 fractures $[16,17,18]$. However, the biomechanical understanding of the radial head as a stabiliser and axial weight-bearing structure led to an appreciation of its functional importance. Excision has become less popular due to concerns about delayed sequelae and improvements in instrumentation for internal fixation and arthroplasty[19]. Also, not all radial head fractures are amenable to simple excision because of concomitant injuries[16]. Complications associated with excision include wrist pain, elbow stiffness, loss of strength, cubitus valgus, synostosis, instability, proximal radial migration, and degenerative arthritis[20]. Broberg and Morrey[21] reported that late excision is equally effective as early excision and may be used as a salvage procedure. Excision can also be considered in patients with isolated, displaced comminuted radial head fractures that are not amenable to fixation[2]. Most comminuted radial head fractures, however, are not isolated[22], and therefore excision alone is often contraindicated.
Choosing to re-establish radiocapitellar mechanics[20] in Mason type 3 fractures by radial head arthroplasty or to preserve the radial head by internal fixation techniques remains controversial. Ring[6] has discussed elbow stability and associated injuries which may be important in determining whether to fix or replace comminuted fractures. As advent of techniques and implants for internal fixation of comminuted radial head fractures developed, it became more popular to attempt to save complex fractures[15]. Some authors have suggested fixation of all comminuted radial fractures, except those with greater than three fragments and where stable fixation may be difficult to achieve[2]. However, combined with increased availability and use of radial head prostheses for comminuted fractures[23,24], the role of fixation is being re-defined. A wide variety of radial head implants have been used since Speed published the first series of ferrule caps for the radial head in 1941[25]. These include acrylic, cobaltchromium, titanium and silicone[26]. Many surgeons believe that it is important to preserve the native radial head, whereas others believe that reliable restoration of radiocapitellar contact with a prosthetic radial head may better address the goals of treatment for comminuted fractures[27]. 
Numerous reviews evaluating fixation versus replacement for Mason type 3 radial head fractures have been published[6,27]. However, none of these have been systematic in terms of search strategy. They all recommend the need for prospective randomised controlled and comparative studies. Therefore in this review, search criteria specifically included comparative studies evaluating fixation versus replacement in adults with Mason type 3 radial head fractures.

\section{Methods}

The Pubmed and Embase databases were searched on 18th June 2014 using keywords and strict eligibility criteria. The studies identified were further limited by selecting "English language articles" only. Duplicate studies were removed. The strategies for these searches are detailed in Tables 1 and 2. Only comparative studies evaluating fixation versus replacement for comminuted (Mason type 3) radial head fractures were included. The critical appraisal checklist (adapted from Critical Appraisal Skills Programme CASP, Oxford)[28,29] and the revised CONSORT checklist[30] for reporting randomised trials were used to guide assessment of the studies identified from the literature search. The full inclusion and exclusion criteria are detailed in Table 3.

Participants: Adults (over 18 years) with Mason type 3 radial head fractures.

Intervention: Surgical fixation with open reduction internal fixation.

Comparator: Radial head replacement.

Outcomes: Primary: functional outcome.

Secondary: complications. 


\begin{tabular}{|c|c|c|c|c|}
\hline & \multicolumn{2}{|l|}{ Search terms } & Medline & Embase \\
\hline 1 & \multicolumn{2}{|l|}{$\exp$ RADIUS FRACTURES/ AND exp ELBOW JOINT/ } & 721 & 366 \\
\hline 2 & \multicolumn{2}{|l|}{$\exp$ RADIUS/ } & 7491 & 9941 \\
\hline 3 & \multicolumn{2}{|l|}{ (radius AND head).ti, ab } & 1572 & 1646 \\
\hline 4 & \multicolumn{2}{|l|}{ "radial head".ti, ab } & 1757 & 1884 \\
\hline 5 & \multicolumn{2}{|l|}{ head.ti,ab } & 223652 & 263240 \\
\hline 6 & \multicolumn{2}{|l|}{2 AND 5} & 874 & 876 \\
\hline 7 & \multicolumn{2}{|l|}{3 OR 4 OR 6} & 3040 & 3241 \\
\hline 8 & \multicolumn{2}{|l|}{ fracture $^{*} \cdot$ ti, ab } & 173942 & 196596 \\
\hline 9 & \multicolumn{2}{|l|}{$7 \mathrm{AND} 8$} & 1326 & 1419 \\
\hline 10 & \multicolumn{2}{|l|}{1 OR 9} & 1668 & 1648 \\
\hline 11 & \multicolumn{2}{|l|}{$\exp$ FRACTURE FIXATION/ } & 46682 & 63420 \\
\hline 12 & \multicolumn{2}{|l|}{ (fixation OR ORIF OR "open reduction internal fixation").ti,ab } & 102644 & 106378 \\
\hline 13 & \multicolumn{2}{|l|}{11 OR 12} & 131425 & 142370 \\
\hline 14 & \multicolumn{2}{|l|}{$\exp$ ARTHROPLASTY/ } & 40292 & 48694 \\
\hline 15 & \multicolumn{2}{|l|}{ (arthroplasty OR replac*).ti,ab } & 334888 & 384221 \\
\hline 16 & \multicolumn{2}{|l|}{14 OR 15} & 345928 & 397571 \\
\hline 17 & \multicolumn{2}{|l|}{10 AND 13 AND 16} & 131 & 151 \\
\hline 18 & \multicolumn{2}{|l|}{17 [Limit to: English Language] } & 105 & 124 \\
\hline 19 & \multicolumn{2}{|c|}{ exp FRACTURES, COMMINUTED/ [Limit to: English Language] } & 1436 & 1934 \\
\hline 20 & \multicolumn{2}{|c|}{$\begin{array}{l}\text { (comminut }{ }^{*} \text { OR multifragment }{ }^{*} \text { OR splinter }{ }^{*} \text { OR crushed OR multi-fragment* OR unreconstructable).ti,ab [Limit to: English } \\
\text { Langtuage] }\end{array}$} & 7409 & 8419 \\
\hline 21 & \multicolumn{2}{|c|}{19 OR 20 [Limit to: English Language] } & 8084 & 9184 \\
\hline 22 & \multicolumn{2}{|l|}{18 AND 21 [Limit to: English Langtuage] } & 38 & 47 \\
\hline \multicolumn{5}{|c|}{ Table 2 Cochrane database search strategy. } \\
\hline 1 & \multicolumn{2}{|c|}{ radial head fracture $:$ ti, ab,kw (word variations have been searched) } & & 30 \\
\hline 2 & \multicolumn{2}{|c|}{ Fixation:ti,ab,kw or ORIF:ti,ab,kw or open reduction internal fixation: ti,ab,kw (word variations have been searched) } & & 4152 \\
\hline 3 & \multicolumn{2}{|c|}{ Arthroplasty:ti,ab,kw or replac*: ti,ab,kw (word variations have been searched) } & & 19668 \\
\hline 4 & $\# 1$ and \#2 and \#3 & & & 7 \\
\hline Tab & le 3 Summary of eligibility criteria. & & & \\
\hline Inc & lusion criteria & Exclusion criteria & & \\
\hline Stu & dies with emphasis on comminuted radial head fractures & Duplicate studies & & \\
\hline & ation/ORIF vs replacement/arthroplasty (comparative studies only) & $\begin{array}{l}\text { Case reports, editorials, comments, letters, guidelines, pr } \\
\text { non-systematic review papers, demographic studies, unp }\end{array}$ & $\begin{array}{l}\text { ocols, abstr } \\
\text { blished stu }\end{array}$ & $\begin{array}{l}\text { acts, } \\
\text { dies }\end{array}$ \\
\hline Pat & ient outcomes data clearly discussed & Anatomical/cadaveric studies & & \\
\hline Ad & ults & Studies investigating only fixation or only replacement & & \\
\hline Eng & glish language articles & & & \\
\hline $\mathrm{Hu}$ & man studies & & & \\
\hline
\end{tabular}




\section{Results}

A total of 92 studies were identified from the literature search (38 Medline, 47 Embase, 7 Cochrane). Of 23 relevant titles 10 were selected for full-text review after assessment of the abstracts. Three articles met inclusion criteria on full-text review
(Figure 1). A summary of these three studies comparing fixation and replacement of Mason type 3 radial head fractures in adults is described in Table 4, and critique of the studies follows on Table 5.

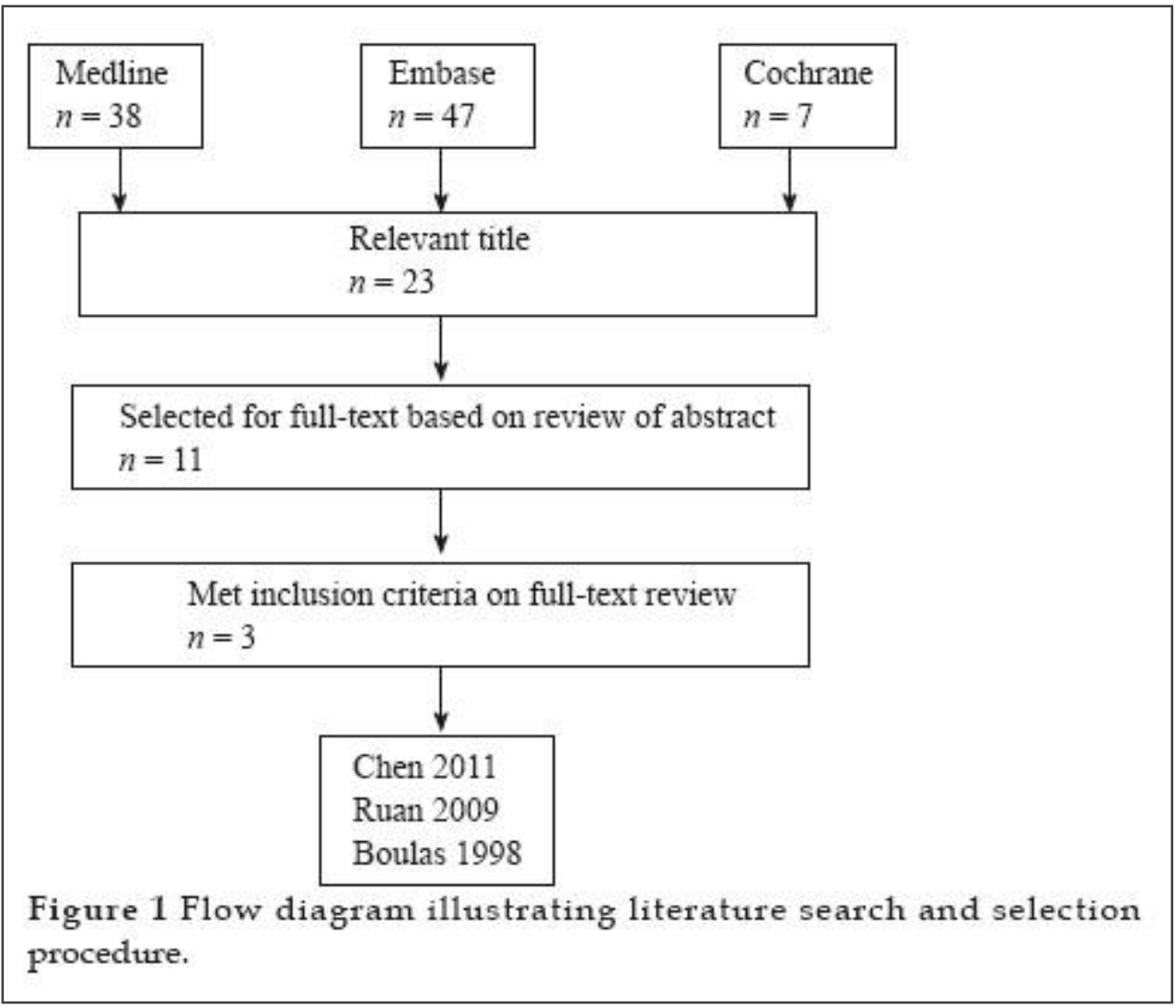




\begin{tabular}{|c|c|c|c|}
\hline Reference & Chen et al, 2011 & Ruan et al, 2009 & Boulas and Morrey, 1998 \\
\hline & $\begin{array}{l}\text { (Comparison between radial head replacement } \\
\text { and open reduction and internal fixation in clinical } \\
\text { treatment of unstable, multi-fragmented radial head } \\
\text { fractures) }\end{array}$ & $\begin{array}{l}\text { (Comparative study of internal } \\
\text { fixation and prosthesis replace- } \\
\text { ment for radial head fractures of } \\
\text { Mason type III) }\end{array}$ & $\begin{array}{l}\text { (Evaluation of the elbow following radial } \\
\text { head fracture. Comparison of ORIF vs. } \\
\text { replacement, excision and non-operative } \\
\text { management) }\end{array}$ \\
\hline Study design" & $\begin{array}{l}\text { Interventional comparative randomised study } \\
\text { Level } 4 \text { evidence } \\
\text { (Stated as "Prospective randomised controlled } \\
\text { study level } 1 \text { evidence" by authors) }\end{array}$ & $\begin{array}{l}\text { Interventional comparative ran- } \\
\text { domised study } \\
\text { Level } 4 \text { evidence }\end{array}$ & $\begin{array}{l}\text { Interventional comparative non-ran- } \\
\text { domised study } \\
\text { Level } 4 \text { evidence }\end{array}$ \\
\hline Research question ${ }^{b}$ & + & $+/-$ & - \\
\hline Sample size & $\begin{array}{l}\text { Total } 45 \text { patients } \\
\text { - } 23 \text { ORIF } \\
\text { - } 22 \text { replacement }\end{array}$ & $\begin{array}{l}\text { Total } 22 \text { patients } \\
-8 \text { ORIF } \\
\text { - } 14 \text { replacement }\end{array}$ & $\begin{array}{l}\text { Total } 36 \text { patients } \\
-7 \text { ORIF } \\
-8 \text { replacement } \\
-13 \text { excision } \\
-8 \text { non-operative }\end{array}$ \\
\hline Participants & $\begin{array}{l}\text { Mean age: } \\
\text { - Combined ORIF/replacement } 37 \text { years } \\
\text { Sex: } \\
\text { - Combined ORIF/replacement } 76 \% \text { male } / 24 \% \\
\text { female } \\
\text { All patients had Mason type } 3 \text { radial head fractures }\end{array}$ & $\begin{array}{l}\text { Mean age: } \\
\text { - ORIF } 40.1 \text { years } \\
\text { - Replacement } 37.4 \text { years } \\
\text { Sex } \\
\text { - ORIF } 5 \text { male } / 3 \text { female } \\
\text { - Replacement } 8 \text { male } / 6 \text { female } \\
\text { All patients had Mason type } 3 \\
\text { radial head fractures }\end{array}$ & $\begin{array}{l}\text { Mean age: } \\
\text { - ORIF } 37.3 \text { years } \\
\text { - Replacement } 48.9 \text { years } \\
\text { Sex: } \\
\text { - ORIF } 3 \text { male } / 4 \text { female } \\
\text { - Replacement } 5 \text { male } / 3 \text { female } \\
\text { Most patients had Mason type } 3 \text { radial } \\
\text { head fractures }\end{array}$ \\
\hline Intervention & $\begin{array}{l}\text { Open reduction and intemal fixation } \\
\text { - AO mini fragment lag screws, plate, Kirshner } \\
\text { wires }\end{array}$ & $\begin{array}{l}\text { Open reduction and internal fixa- } \\
\text { tion } \\
\text { - cannulated screws, Kirschner } \\
\text { wires }\end{array}$ & $\begin{array}{l}\text { Open reduction and internal fixation } \\
\text { - AO muni fragment lag screws, buttress } \\
\text { plate, Herbert screws }\end{array}$ \\
\hline Comparator & $\begin{array}{l}\text { Radial head replacement } \\
\text { - Monopolar titanium prosthesis }\end{array}$ & $\begin{array}{l}\text { Radial head replacement } \\
\text { - Cement stem and bipolar pros- } \\
\text { thesis }\end{array}$ & $\begin{array}{l}\text { Radial head replacement } \\
\text { - Silastic prosthesis }\end{array}$ \\
\hline Observer blinding" & + (randomuised block design) & - & - \\
\hline Follow-up & $\begin{array}{l}\text { Combined ORIF/replacement - } 26 \text { months } \\
\text { (range } 21 \text { - } 37 \text { months) } \\
\text { (not split into ORIF/replacement) }\end{array}$ & $\begin{array}{l}\text { ORIF group - } 14 \text { months } \\
\text { (range } 10-21 \text { months) } \\
\text { Replacement group }-15.9 \text { months } \\
\text { (range } 10-27 \text { months) }\end{array}$ & $\begin{array}{l}\text { ORIF group - } 36 \text { months } \\
\text { (range } 14-64 \text { months) } \\
\text { Replacement group - } 49 \text { months } \\
\text { (range } 12-96 \text { months) }\end{array}$ \\
\hline $\begin{array}{l}\text { Removal of prosthe } \\
\text { ses }\end{array}$ & $\begin{array}{l}\text { ORIF (19) reason not stated } \\
\text { Replacement - }\end{array}$ & $\begin{array}{l}\text { ORIF (2) removed for metalwork } \\
\text { irritation } \\
\text { Replacement - }\end{array}$ & - \\
\hline Primary outcomes & $\begin{array}{l}\text { Broberg and Morrey functional elbow criteria } \\
\text { ORIF - excellent (9), good ( } 6 \text { ), fair (5), poor ( } 3 \text { ) } \\
\text { Replacement - Excellent (15), good (5), fair (1), poor } \\
\text { (1) } \\
\text { Good/ Excellent result in } 65.2 \% \text { ORIF group com- } \\
\text { pared to } 91 \% \text { replacement group } \\
\text { Statistically significant difference ( } P<0.01 \text {; t-test } \\
\text { and chi-squared test) }\end{array}$ & $\begin{array}{l}\text { Broberg and Morrey functional } \\
\text { elbow criteria: } \\
\text { ORIF - good (1), fair ( } 4 \text { ), poor ( } 3 \text { ) } \\
\text { Replacement - Excellent ( } 9 \text { ), good } \\
\text { (4), fair (1) } \\
\text { Good/ Excellent result in } 12.5 \% \\
\text { ORIF group compared to } 92.9 \% \\
\text { replacement group } \\
\text { Statistically significant difference } \\
\text { ( } P=0.0004 \text {; Fisher's exact test) }\end{array}$ & $\begin{array}{l}\text { Mayo performance index (max score = } \\
100 \text { ): } \\
\text { ORIF - mean } 97.9 \\
\text { (range } 85-100 \text { ) } \\
\text { Replacement - mean } 94.4 \\
\text { (range } 85-100 \text { ) } \\
\text { No significant difference in scores } \\
\text { Motion at last follow-up: } \\
\text { ORIF group mean - ext } 10^{\circ} \text {, flex } 146^{\circ} \text {, } \\
\text { pron } 70^{\circ} \text {, sup } 66^{\circ} \\
\text { Replacement group mean- ext } 11^{\circ} \text {, flex } \\
145^{\circ} \text {, pron } 55^{\circ} \text {, sup } 79^{\circ} \\
\text { No significant difference in range of mo- } \\
\text { tion ( }(P<0.05 \text { ) } \\
\text { Strength (conrected for hand donui- } \\
\text { nance): } \\
\text { - Grip strength significantly higher in } \\
\text { ORIF group }(P<0.05 \text { ), no difference in } \\
\text { other strength parameters }\end{array}$ \\
\hline Secondary outcomes & $\begin{array}{l}\text { Complications } \\
\text { ORIF - non-union (1), range of motion deficit }>30^{\circ} \\
\text { (4), fixation failure and fragment displacement ( } 3 \text {, } \\
\text { deep wound infection (1), heterotopic ossification } \\
\text { (2) } \\
\text { Replacement - range of motion deficit }>30^{\circ}(2) \text {. } \\
\text { joint stiffness (1) } \\
\text { Statistically significant difference in complication } \\
\text { rates }(P<0.01)\end{array}$ & $\begin{array}{l}\text { Complications }{ }^{d} \text { : } \\
\text { ORIF group - non-union and k- } \\
\text { wire loosening (4) } \\
\text { Replacement group - heterotopic } \\
\text { ossification (3) }\end{array}$ & $\begin{array}{l}\text { Complications } \\
\text { ORIF group - removal of metalwork due } \\
\text { to irritation (2) } \\
\text { Replacement group' - heterotopic os- } \\
\text { sification ( } 4 \text { ). prosthesis dislocation (1). } \\
\text { breakage of prosthesis (1) }\end{array}$ \\
\hline \multicolumn{4}{|c|}{$\begin{array}{l}\text { "Based on modified Oxford Centre for Evidence-based medicine - Levels of Evidence (March } 2009) 34^{b}(+) \text { Well defined, in introduction; }(+/-) \text { well define } \\
\text { after reading article; }(-) \text { not well defined; }(+) \text { complete; }(+/-) \text { incomplete; }(-) \text { none; "numbers in brackets indicate number of patients; }(+) \text { described and pe } \\
\text { formed; }(+/-) \text { described, not performed; }(-) \text { not described; "Morrey ct al } 35 \text { (see references) }\end{array}$} \\
\hline
\end{tabular}




\begin{tabular}{|c|c|c|c|c|c|c|c|c|c|c|}
\hline Study & $\begin{array}{l}\text { Clear } \\
\text { state- } \\
\text { ment } \\
\text { of } \\
\text { aims? }\end{array}$ & $\begin{array}{l}\text { Is methodol- } \\
\text { ogy appropri- } \\
\text { ate? }\end{array}$ & $\begin{array}{l}\text { Design } \\
\text { appropri- } \\
\text { ate to } \\
\text { address } \\
\text { aims? }\end{array}$ & $\begin{array}{l}\text { Appropriate recruit- } \\
\text { ment strategy? }\end{array}$ & $\begin{array}{l}\text { Appropriate data collec- } \\
\text { tion? }\end{array}$ & $\begin{array}{l}\text { Appropriate } \\
\text { consideration } \\
\text { of researcher/ } \\
\text { participant } \\
\text { role? }\end{array}$ & $\begin{array}{l}\text { Ethical } \\
\text { issues } \\
\text { consid- } \\
\text { ered? }\end{array}$ & $\begin{array}{l}\text { Suf- } \\
\text { ficiently } \\
\text { rigorous } \\
\text { data } \\
\text { analysis? }\end{array}$ & $\begin{array}{l}\text { Clear } \\
\text { state- } \\
\text { ment of } \\
\text { find- } \\
\text { ings? }\end{array}$ & $\begin{array}{l}\text { How valu- } \\
\text { able is } \\
\text { research? }\end{array}$ \\
\hline $\begin{array}{l}\text { Chen } \\
\text { ct al }\end{array}$ & Yes & $\begin{array}{l}\text { Yes but study } \\
\text { stated as } \\
\text { "Prospective } \\
\text { randomised } \\
\text { controlled } \\
\text { study level" by } \\
\text { authors - on } \\
\text { closer reading } \\
\text { study better } \\
\text { classified as } \\
\text { level } 4 \text { com- } \\
\text { parative trial }\end{array}$ & $\begin{array}{l}\text { Yes but } \\
\text { design not } \\
\text { clearly } \\
\text { explained } \\
\text { Patient- } \\
\text { assessed, } \\
\text { elbow- } \\
\text { specific } \\
\text { outcome } \\
\text { score used }\end{array}$ & $\begin{array}{l}\text { Clear inclusion/ex- } \\
\text { clusion criteria } \\
\text { No reference made } \\
\text { to number of fracture } \\
\text { fragments, stabil- } \\
\text { ity and additional } \\
\text { injuries } \\
\text { Randomisation } \\
\text { method for allocation } \\
\text { of groups not stated } \\
\text { No mention of al- } \\
\text { location concealment } \\
\text { Baseline demograph- } \\
\text { ics and clinical char- } \\
\text { acteristics of pts not } \\
\text { adequately discussed } \\
\text { Non-matched } \\
\text { groups }\end{array}$ & $\begin{array}{l}\text { Prospective randomised } \\
\text { controlled study } \\
\text { Surgical technique of both } \\
\text { methods stated but deci- } \\
\text { sion for choice of treatment } \\
\text { methods within ORIF } \\
\text { group not explained } \\
\text { Clear post-operative reha- } \\
\text { bilitation regime stated } \\
\text { No sample size calculation } \\
\text { performed } \\
\text { No outcome measures } \\
\text { stated in methods section, } \\
\text { only in results section } \\
\text { Setting and location of data } \\
\text { collection not stated } \\
\text { Follow-up period clearly } \\
\text { stated } \\
\text { Radiographic parameters } \\
\text { clearly defined }\end{array}$ & $\begin{array}{l}\text { Grade of } \\
\text { surgeon not } \\
\text { stated } \\
\text { Reviewers } \\
\text { blinded by } \\
\text { randomised } \\
\text { block design } \\
\text { All clinical } \\
\text { assessments } \\
\text { performed by } \\
\text { independent } \\
\text { observers } \\
\end{array}$ & $\begin{array}{l}\text { Ap- } \\
\text { proved } \\
\text { by } \\
\text { authors' } \\
\text { institu- } \\
\text { tional } \\
\text { ethics } \\
\text { commuit- } \\
\text { tee }\end{array}$ & $\begin{array}{l}\text { Appro- } \\
\text { priate } \\
\text { statistical } \\
\text { methods } \\
\text { No power } \\
\text { calcula- } \\
\text { tion } \\
\text { No con- } \\
\text { fidence } \\
\text { intervals } \\
\text { calcu- } \\
\text { lated }\end{array}$ & $\begin{array}{l}\text { State- } \\
\text { ment of } \\
\text { findings } \\
\text { vague } \\
\end{array}$ & $\begin{array}{l}\text { Study linked } \\
\text { to current } \\
\text { knowledge/ } \\
\text { trends } \\
\text { No mention } \\
\text { of further } \\
\text { work } \\
\text { Relevant } \\
\text { literature } \\
\text { review con- } \\
\text { ducted }\end{array}$ \\
\hline $\begin{array}{l}\text { Ruan } \\
\text { et al }\end{array}$ & Yes & Yes & $\begin{array}{l}\text { Yes but } \\
\text { design not } \\
\text { clearly } \\
\text { explained } \\
\text { Patient- } \\
\text { assessed, } \\
\text { elbow- } \\
\text { specific } \\
\text { outcome } \\
\text { score used }\end{array}$ & $\begin{array}{l}\text { Inclusion/exclusion } \\
\text { criteria not clearly } \\
\text { stated } \\
\text { No reference made } \\
\text { to number of fracture } \\
\text { fragments, stabil- } \\
\text { ity and additional } \\
\text { injuries } \\
\text { Randomisation } \\
\text { method for allocation } \\
\text { of groups not stated } \\
\text { No mention of al- } \\
\text { location concealment } \\
\text { Baseline demo- } \\
\text { graphics of pts not } \\
\text { adequately discussed } \\
\text { Non-matched groups } \\
\text { (2 old fractures in } \\
\text { replacement group) }\end{array}$ & $\begin{array}{l}\text { Comparative study } \\
\text { Replacement technique } \\
\text { stated clearly, ORIF tech- } \\
\text { nique stated very briefly } \\
\text { Rehab regime stated but no } \\
\text { details } \\
\text { No sample size calculation } \\
\text { performed } \\
\text { No outcome measures } \\
\text { stated in methods section, } \\
\text { only in results section } \\
\text { Setting and location of data } \\
\text { collection not stated } \\
\text { Follow-up period clearly } \\
\text { stated }\end{array}$ & $\begin{array}{l}\text { Number } \\
\text { and grade of } \\
\text { surgeon(s) } \\
\text { not stated } \\
\text { No mention } \\
\text { of observer } \\
\text { blinding }\end{array}$ & $\begin{array}{l}\text { Not } \\
\text { stated } \\
\text { Con- } \\
\text { flicts of } \\
\text { interest } \\
\text { and } \\
\text { sources } \\
\text { of fund- } \\
\text { ing not } \\
\text { stated }\end{array}$ & $\begin{array}{l}\text { Appro- } \\
\text { priate } \\
\text { statistical } \\
\text { methods } \\
\text { No power } \\
\text { calcula- } \\
\text { tion } \\
\text { No con- } \\
\text { fidence } \\
\text { intervals } \\
\text { calcu- } \\
\text { lated }\end{array}$ & $\begin{array}{l}\text { State- } \\
\text { ment of } \\
\text { findings } \\
\text { vague }\end{array}$ & $\begin{array}{l}\text { Study linked } \\
\text { to current } \\
\text { knowledge/ } \\
\text { trends } \\
\text { Brief sug- } \\
\text { gestion of } \\
\text { further work } \\
\text { Relevant } \\
\text { literature } \\
\text { review con- } \\
\text { ducted }\end{array}$ \\
\hline $\begin{array}{l}\text { Boulas } \\
\text { and } \\
\text { Mor- } \\
\text { rey }\end{array}$ & Yes & Yes & $\begin{array}{l}\text { Yes but } \\
\text { designnot } \\
\text { clearly } \\
\text { explained } \\
\text { Clini- } \\
\text { cian- and } \\
\text { patient- } \\
\text { assessed, } \\
\text { ellow- } \\
\text { specific } \\
\text { outcome } \\
\text { score used }\end{array}$ & $\begin{array}{l}\text { Vague inclusion } \\
\text { criteria, exclusion } \\
\text { criteria not stated } \\
\text { No reference made } \\
\text { to number of fracture } \\
\text { fragments, stabil- } \\
\text { ity and additional } \\
\text { injuries } \\
\text { No mention of ran- } \\
\text { domisation } \\
\text { Baseline demograph- } \\
\text { ics and clinical char- } \\
\text { acteristics of pts not } \\
\text { adequately discussed } \\
\text { Non-matched } \\
\text { groups } \\
\text { Fractures heteroge- } \\
\text { neous (not limited to } \\
\text { Mason type 3), exact } \\
\text { number of Mason } \\
\text { type } 3 \text { not stated }\end{array}$ & $\begin{array}{l}\text { Retrospective comparative } \\
\text { study } \\
\text { Surgical method or details } \\
\text { of implants not adequately } \\
\text { explained } \\
\text { Decision for choice of } \\
\text { treatment methods not } \\
\text { explained } \\
\text { No sample size calculation } \\
\text { performed } \\
\text { Setting and location of data } \\
\text { collection not stated } \\
\text { Rehab regime not stated } \\
\text { Mean follow-up period } \\
\text { stated } \\
\text { Radiographic findings } \\
\text { clearly defined }\end{array}$ & $\begin{array}{l}\text { Number } \\
\text { and grade of } \\
\text { surgeon(s) } \\
\text { not stated } \\
\text { No mention } \\
\text { of observer } \\
\text { blinding } \\
\text { Radiographic } \\
\text { findings not } \\
\text { independent- } \\
\text { ly verified }\end{array}$ & $\begin{array}{l}\text { Not } \\
\text { stated } \\
\text { Con- } \\
\text { flicts of } \\
\text { interest } \\
\text { and } \\
\text { sources } \\
\text { of fund- } \\
\text { ing not } \\
\text { stated }\end{array}$ & $\begin{array}{l}\text { Appro- } \\
\text { priate } \\
\text { statistical } \\
\text { methods } \\
\text { Factorial } \\
\text { analy- } \\
\text { sis of } \\
\text { variance } \\
\text { allowing } \\
\text { multiple } \\
\text { compari- } \\
\text { son used } \\
\text { No power } \\
\text { calcula- } \\
\text { tion } \\
\text { No con- } \\
\text { fidence } \\
\text { intervals } \\
\text { calcu- } \\
\text { lated }\end{array}$ & $\begin{array}{l}\text { Clear } \\
\text { state- } \\
\text { ment of } \\
\text { findings }\end{array}$ & $\begin{array}{l}\text { Study linked } \\
\text { to current } \\
\text { knowledge/ } \\
\text { trends } \\
\text { Basic review } \\
\text { of literature } \\
\text { conducted }\end{array}$ \\
\hline
\end{tabular}




\section{Discussion}

The purpose of this systematic review was to determine whether fixation or replacement provides better functional outcomes for Mason type 3 radial head fractures. Chen et al[31] concluded that metallic radial head replacement gave better joint function that ORIF. Ruan et al[32] concluded that bipolar radial head replacement was better than ORIF. Boulas[33] concluded that, when possible, ORIF provided better functional results than silastic replacement.

A number of methodological deficiencies were found in these studies. These included: small sample sizes; limited follow-up period; no controlling for confounding factors; no power calculation to determine probability of type II error; no observer blinding.

The small sample sizes in the studies may indicate selection bias, reporting bias, or both. The varying follow-up might indicate a significant amount of "missing" events and complications occurring after the follow-up period. Surgical methods were different in all three papers and no clear explanations were given for the types of fixation chosen. Fixation can be obtained with various implants, the goal being stable articular surface fixation and restoration of the head-neck relationship[2]. Currently, fixation has become popular, since contemporary techniques have improved surgical outcomes[15,36]. Good surgical outcomes have been shown in selected Mason type 3 radial head fractures and fracture dislocations stabilized with internal fixation[37]. Complications of fixation include metalwork failure, irritation, non-union and poor forearm motion.

Most radial head implants in use today are metal, consisting of cobalt-chrome or titanium. These have been found to restore axial and valgus stability, whereas silicone implants cannot[26]. Comparative clinical evidence supporting a particular design concept for radial head arthroplasty, such as bipolar versus monopolar, cemented versus uncemented, and anatomic versus asymmetric head shape is not yet available[2]. However, good to excellent results can be anticipated when radial head replacement is used for the correct indications and when care is taken to understand concomitant injuries[25]. Recent clinical outcome studies of metallic radial head arthroplasty systems indicate it is a reasonable option to offer patients with comminuted radial head fractures[19]. Specific complications include synostosis 
formation, heterotopic ossification, loosening and stiffness[26].

The study by Boulas[33] compared fixation to silastic replacement. Evidence has shown that silicone radial head implants have multiple complications[38,39]. These include implant fractures and silicone synovitis. These complications and biomechanical studies showing the inability of silicone implants to support the radius functionally have lead to their disuse[19]. However recently other authors have argued that the silastic implant can be used in a stable elbow with good functional and radiographic results[40]. Therefore Boulas' paper[33] has been included in this systematic review. The inclusion of this study could be seen to be a limitation of this systematic review.

Important factors in considering operative treatment for Mason type 3 radial head fractures include elbow stability, associated injuries and number of fragments. None of the studies identified in this systematic review gave this important baseline patient information. Recent studies have discussed the principles that guide decision-making in the management of comminuted radial head fractures[2,22,27]. Pike et al[2] recommended ORIF when feasible for all displaced $(>2 \mathrm{~mm}$ ) radial head fractures consisting of less than four fragments. They recommended radial head arthroplasty if ORIF was not feasible and in the presence of elbow instability or if greater then three fragments were present. Clembosky and Borretto[27] attempted to repair all comminuted radial head fractures, as they concluded radial head arthroplasty could not restore valgus stability when the medial collateral ligament was deficient. However, they stated that if stable fixation could not be achieved, there was a definite risk of early failure and non-union. They therefore recommended arthroplasty in these complicated comminuted fractures as it produced consistent results and was more straightforward than fixation. Considering these reviews, it is evident that the lack of detail regarding baseline characteristics of patients in the studies by Chen[31], Ruan[32] and Boulas[33] is a major weakness. This is another limitation of this search strategy.

There are other factors that may have affected the findings of this systematic review. Firstly, only English language articles were included and this may have limited the breadth of literature review. Secondly, the search strategy was based on a computer search process. Computer searches may omit some 
articles, and consequently, limit the scope of the literature search[41].

In conclusion, in our systematic review two studies found significantly better Broberg and Morrey functional scores after replacement compare with ORIF for Mason type 3 radial head fractures. The third study found no significant difference in functional score (Mayo performance index) or range of motion, but did find that grip strength was better after ORIF. Complication rates were too heterogenous for conclusion. Considering the small numbers of studies, limitations of the search strategy and methodological weaknesses within the studies included in this systematic review, it is not definitively possible to clarify whether fixation or replacement has better functional outcomes in patients with Mason type 3 radial head fractures. As discussed, the principles that guide decision making in the treatment of Mason type 3 radial head fractures include elbow stability and degree of comminution. Each individual case should take these factors into account and only then a decision on fixation or replacement should be made. To help clarify the decision making, randomised studies comparing the two treatment methods and taking stability into account are needed. This may be difficult as many unstable, comminuted fractures are difficult or impossible to fix[6]. Future studies should also compare the various types of radial head implants in randomised comparative studies. Also, better data is required regarding the long-term outcome of metal radial head replacements.

\section{Reference}

1 Morrey B. Radial head fracture. In: Morrey B, editor. The elbow and its disorders. 3rd ed. Philadelphia: WB Saunders, 2000: 341-364. ISBN 0721677525, 9780721677521.

2 Pike J, Athwal G, Faber M, King G. Radial head fractures - an update. J Hand Surg 2009; 34A: 557565. doi: 10.1016/j.jhsa.2008.12.024.

3 Rosenblatt Y, Athwal G, Faber $K$. Current recommendations for the treatment of radial head fractures. Orthop Clin N Am 2008; 39: 173-185. doi: 10.1016/j.ocl.2007.12.008.

4 Harrington I, Tountas A. Replacement of the radial head in 
the treatment of unstable unstable elbow fractures. Injury 1981; 12: 405412.

5 Harrison J, Chitre A, Lammin K, Warner J, Hodgson S. Radial head fractures in adults. Current Orthopedics 2007; 21: 59-64. http://dx.doi.org/10.1016/j.cuor.20 06.10 .003

6 Ring D. Displaced, unstable fractures of the radial head: Fixation vs replacement - What is the evidence? Injury 2008; 39: 1329-1337. doi: 10.1016/j.injury.2008.04.011

7 Mason M. Some observations on fracture of the head of the radius with review of one hundred cases. $\mathrm{Br}$ J Surg 1954; 42: 123-132.

8 Johnston G. A follow-up of one hundred cases of fracture of the head of the radius with a review of the literature. Ulster Med J 1962; 31: 51-56.

9 Broberg M, Morrey B. Results of treatment of fracture dislocations of the elbow. Clin Orthop Rel Res 1987: 109-119.

10 Hotchkiss R. Displaced fractures of the radial head: internal fixation or excision? J Am Acad Orthop Surg 1997; 5: 1-10.

11 Schatzker J. Fractures of the radial head. In Schatzker J, Tile M, eds. The rationale of operative fracture care. 2nd ed. SpringerVerlag, 1996: 131-135. ISBN 3540593888, 9783540593881.

12 Roidis N, Papadakis S, Rigopoulos N, Basdekis G, Poulsides
L, Karachalios T, Malizos K, Itamura J. Current concepts and controversies in the management of radial head fractures. Orthopedics 2006; 29(10): 904-916.

13 Sharpe F, Kuschner S. Radial head fractures. In: Baker C, Plancher $\mathrm{K}$, editors. Operative treatment of elbow injuries. NewYork, NY: Springer-Verlag Inc, 2001: 207-233. ISBN 0387989056, 9780387989051

14 Akesson $\mathrm{T}$, Herbertsson $\mathrm{P}$, Josefsson P-O, Hasserius R, Besjakov J, Karlsson M. Primary nonoperative treatment of moderately displaced two-part fractures of the radial head. J Bone Joint Surg Am, 2006; 88(9): 1909-1914. doi: 10.2106/JBJS.E.01052.

15 Ring D, Quintero J, Jupiter J. Open reduction and internal fixation of fractures of the radial head. J Bone Joint Surg Am. 2002; 84: 1811-1815.

16 Furry K, Clinkscales C. Comminuted fractures of the radial head: arthroplasty versus internal fixation. Clin Orth Rel Res 1998; 353: 40-52.

17 Hotchkiss R. Fractures and dislocations of the elbow. In Rockwood C, Green D, editors. Fractures in adults. 4th ed. Philadelphia, PA: Lippincott-Raven, 1996: 929-1024. ISBN 039751509X, 9780397515097.

18 McKee M, Jupiter J. Trauma to the adult elbow and fractures of the distal humerus. In: Browner B, Jupiter J, Levine A, Trafton P, editors. Skeletal trauma. 2nd ed. 
Philadelphia, PA: WB Saunders, 1998: 1455-1522. ISBN 0721668844, 9780721668840

19 Calfee R, Madom I, Weiss R. Radial head arthroplasty. J Hand Surg 2006; 31A: 314-321. doi: 10.1016/j.jhsa.2005.12.005.

20 Morrey B, An K, Stormont T. Force transmission through the radial head. J Bone Joint Surg 1988; 70A: 250-256.

21 Broberg M, Morrey B. Results of delayed excision of the radial head after fracture. J Bone Joint Surg Am. 1986; 68: 669-674.

22 Riet R, Morrey B. Documentation of associated injuries occurring with radial head fracture. Clin Orthop Relat Res 2008; 466: 130134. doi: 10.1007/s11999-007-0064-8.

23 Knight D, Rymaszewski L, Amis A, Miller J. Primary replacement of the fractured radial head with a metal prosthesis. J Bone Joint Surg 1993; 75B: 572-576.

24 Moro J, Werier J, MacDermid J. Arthroplasty with a metal radial head for unreconstructable fractures of the radial head. J Bone Joint Surg 2001; 83A: 1201-1211.

25 Speed K. Ferrule caps for the head of the radius. Surg Gynecol Obstet 1941; 73: 845.

26 Stuffman E, Baratz M. Radial head implant arthroplasty. J Hand Surgery 2009; 34A: 745-754. doi: 10.1016/j.jhsa.2009.01.027.

27 Clembosky G, Boretto J. Open reduction and internal fixation versus prosthetic replacement for complex fractures of the radial head. J Hand Surg 2009; 34(6): 1120-1123. doi: 10.1016/j.jhsa.2008.12.031.

28 Guyatt G, Sackett D, Cook D. Users' guides to the medical literature. How to use an article about therapy or prevention. A. Are the results of the study valid? JAMA 1993; 270: 2598-2601.

29 Guyatt G, Sackett D, Cook D. Users' guides to the medical literature. How to use an article about therapy or prevention. B. What were the results and will they help me in caring for my patients? JAMA 1993; 271: 59-63.

30 Schulz K, Altman D, Moher D. CONSORT 2010 Statement: updated guidelines for reporting parallel group randomised trials. BMJ 2010; 340: c869. doi: 10.1136/bmj.c869.

31 Chen X, Wang S, Cao L, Yang G, Li M, Su J. Comparison between radial head replacement and open reduction and internal fixation in clinical treatment of unstable, multifragmented radial head fractures. International Orthopaedics (SICOT) 2011; 35: 1071-1076. doi: 10.1007/s00264-010-1107-4.

32 Ruan H, Fan C, Liu J, Zeng B. A comparative study of internal fixation and prosthesis replacement for radial head fractures of Mason type III. International Orthopaedics 2009; 33: 249-253. doi: 10.1007/ s00264-007-0453-3 
33 Boulas H, Morrey B. elbow dislocation. J Hand Surg 2007; Evaluation of the elbow following 32A: $\quad 1560-1568 . \quad$ doi: 10.1016/j.jhsa.2007.09.016

38 Swanson A, Jaeger S, La open reduction and internal fixation vs. replacement, excision and nonoperative management. Ann Hand Surg 1998; 17(4): 314-320.

34 No author named. Oxford Centre for Evidence-based Medicine - Levels of Evidence (March 2009). http://www.cebm.net/oxfordcentre-evidence-based-medicinelevels-evidence-march-2009/ (accessed 19 January 2015)

35 Morrey B, Askew L, Chao E. Silastic prosthetic replacement for the radial head. J Bone Joint Surg 1981; 63A: 454-458.

36 McArthur R. Herbert screw fixation of fracture of the head of the radius. Clin Orthop Relat Res 1987; 224: 79-87.

37 Nalbantoglu U, Kocaoglu B, Gereli A, Aktas S, Guven O. Open reduction and internal fixation of Mason type III radial head fractures with and without an associated 